\title{
Poultry: the most common food in outbreaks with known pathogens, United States, 1998-2012
}

\author{
S. J. CHAI, D. COLE, A. NISLER AND B. E. MAHON* \\ Enteric Diseases Epidemiology Branch; Division of Foodborne, Waterborne, and Environmental Diseases; \\ Centers for Disease Control and Prevention, Atlanta, GA, USA
}

Received 14 May 2016; Final revision 19 September 2016; Accepted 19 September 2016; first published online 26 October 2016

\section{SUMMARY}

As poultry consumption continues to increase worldwide, and as the United States accounts for about one-third of all poultry exports globally, understanding factors leading to poultryassociated foodborne outbreaks in the United States has important implications for food safety. We analysed outbreaks reported to the United States' Foodborne Disease Outbreak Surveillance System from 1998 to 2012 in which the implicated food or ingredient could be assigned to one food category. Of 1114 outbreaks, poultry was associated with $279(25 \%)$, accounting for the highest number of outbreaks, illnesses, and hospitalizations, and the second highest number of deaths. Of the 149 poultry-associated outbreaks caused by a confirmed pathogen, Salmonella enterica $(43 \%)$ and Clostridium perfringens $(26 \%)$ were the most common pathogens. Restaurants were the most commonly reported location of food preparation ( $37 \%$ of poultry-associated outbreaks), followed by private homes $(25 \%)$, and catering facilities $(13 \%)$. The most commonly reported factors contributing to poultry-associated outbreaks were food-handling errors $(64 \%)$ and inadequate cooking (53\%). Effective measures to reduce poultry contamination, promote safe food-handling practices, and ensure food handlers do not work while ill could reduce poultry-associated outbreaks and illnesses.

Key words: Foodborne infections, food safety, outbreaks.

\section{INTRODUCTION}

Poultry is the second-most commonly consumed meat worldwide, and consumption of poultry is projected to increase more quickly than any other major meat [1]. The United States is one of the top two poultry suppliers worldwide, accounting for about one-third of all poultry exports during 2011-2014. Similar to worldwide trends, poultry is an important and increasing

\footnotetext{
* Author for correspondence: Dr B. E. Mahon, Centers for Disease Control and Prevention, 1600 Clifton Road, MS C-09, Atlanta, GA 30333, USA.

(Email: bdm3@cdc.gov)
}

part of the US diet. Per capita consumption doubled during 1970-2007, and poultry accounted for almost a quarter of meat consumed in the United States in 2007 [2]. Therefore, understanding the contribution of poultry to foodborne outbreaks in the United States is important not only for the United States but also globally. Foodborne outbreak investigations provide the most readily available information about the sources and circumstances of food contamination leading to illness.

In the United States, consumption of food contaminated with known pathogens causes an estimated $9 \cdot 4$ million illnesses every year [3]. Poultry is an important source of these foodborne infections [4-7]. Poultry 
products are contaminated frequently with some of the most common bacterial pathogens associated with foodborne illness [8]. In 2011, cultures of $13 \%$ of chicken samples and $6 \%$ of ground turkey samples yielded Salmonella, and $38 \%$ of chicken samples yielded Campylobacter [8].

Large multistate outbreaks associated with poultry have focused industry, regulatory, and consumer attention on the safety of poultry products. For example, in 2013-2014, a 29-state outbreak of multidrug-resistant Salmonella enterica serotype Heidelberg infections associated with chicken caused 634 illnesses and 241 hospitalizations [9]. We conducted a detailed analysis of the pathogens, settings, contamination factors, and poultry types leading to poultry-associated foodborne outbreaks in the United States to guide interventions to decrease illnesses associated with poultry consumption.

\section{METHODS}

\section{Data source}

The Foodborne Disease Outbreak Surveillance System (FDOSS) collects reports of foodborne disease outbreaks from state, local, and territorial public health agencies in the United States. The Centers for Disease Control and Prevention (CDC) runs FDOSS, and defines a foodborne disease outbreak as the occurrence of two or more similar illnesses resulting from ingestion of the same food. Reported data include the number of illnesses, hospitalizations, and deaths; the pathogen; the implicated food(s); and the settings of food preparation and consumption. FDOSS also collects information regarding certain contributing factors (e.g. food not adequately cooked or not kept at the right temperature before consumption) [10]. Demographic data are reported as percentages of ill persons in defined age and gender categories. Pathogens are reported as confirmed if specific diagnostic criteria were met [11]. During the period of this study, 1998-2012, FDOSS classified foods into 17 categories (fish, crustaceans, molluscs, dairy, eggs, beef, game, pork, poultry, grains-beans, oils-sugars, fruits-nuts, fungi, leafy, root, sprout, and vine-stalk) [12].

\section{Analysis}

We included outbreaks satisfying the following criteria: (1) the outbreak occurred from 1998 to 2012,
(2) the investigation implicated a food using either statistical or laboratory evidence, (3) the food contained a single contaminated ingredient or multiple contaminated ingredients belonging to a single food category, and (4) a suspected or confirmed pathogen was reported. We conducted three analyses. First, we examined all outbreaks with a confirmed or suspected pathogen in which investigators implicated poultry and contrasted the number of illnesses, hospitalizations, and deaths with outbreaks caused by other food categories. Second, we examined poultry-associated outbreaks with a confirmed pathogen by pathogen. Third, we compared poultry-associated outbreaks with a confirmed pathogen by type of poultry implicated. For outbreaks with demographic information reported, we estimated the number of ill persons in defined age and sex categories by multiplying the reported percentage in the category by the reported number of ill persons in the outbreak.

Reports could contain one or more contributing factors for each outbreak. We classified these factors into broad categories, including food-handling errors, inadequate cooking, contamination before food preparation, contaminated equipment, and poor personal hygiene of a food handler (Supplementary Table S1).

For statistical analysis, we assumed that the outbreaks included in our analysis represented a random sample of foodborne outbreaks occurring in the United States during the study period. We evaluated categorical variables using frequencies and using a $\chi^{2}$ test (Fisher's exact test for comparisons with cell counts <5) for comparisons. We used one-way fixed-effects analysis of variance to compare means of counts of illnesses between poultry and non-poultry outbreaks and between chicken and turkey outbreaks. We used the Wilcoxon rank sum test to compare distributions of percentages of demographic characteristic variables that were not normally distributed. We used a cut-off of $\alpha=0.05$ to indicate statistical significance and conducted analyses using SAS v. 9.3 (SAS Institute, USA).

\section{RESULTS}

\section{All outbreaks with a confirmed or suspected pathogen}

During 1998-2012, FDOSS received 1114 reports of foodborne disease outbreaks that met the inclusion criteria. Poultry was the most frequently reported food category ( 279 outbreaks, $25 \%$ ), followed by fish $(181,16 \%)$ and beef $(135,12 \%)$. The 279 outbreaks 
Table 1. Reported foodborne disease outbreaks with a confirmed pathogen associated with poultry and with foods other than poultry*, by pathogen, United States, 1998-2012

\begin{tabular}{|c|c|c|c|c|c|}
\hline \multirow[b]{2}{*}{ Pathogen } & \multicolumn{4}{|c|}{ Poultry, $n(\%)$} & \multirow{2}{*}{$\begin{array}{l}\text { Foods other } \\
\text { than poultry } \\
n(\%) \\
\text { Total }\end{array}$} \\
\hline & Chicken & Turkey & Other poultry & Total & \\
\hline Salmonella enterica & $39(41)$ & $25(48)$ & $0(0)$ & $64(43)$ & $160(40)$ \\
\hline Clostridium perfringens & $23(24)$ & $16(31)$ & $0(0)$ & $39(26)$ & $67(17)$ \\
\hline Campylobacter spp. & $7(7)$ & $2(4)$ & $1(33)$ & $10(7)$ & $37(9)$ \\
\hline Norovirus & $6(6)$ & $3(6)$ & $1(33)$ & $10(7)$ & $56(14)$ \\
\hline Staphylococcus aureus & 7 (7) & $1(2)$ & $0(0)$ & $8(5)$ & $43(11)$ \\
\hline Listeria monocytogenes & $1(1)$ & $4(8)$ & $0(0)$ & $5(3)$ & $10(3)$ \\
\hline Bacillus cereus & $4(4)$ & $0(0)$ & $0(0)$ & $4(3)$ & $17(4)$ \\
\hline Othert & $7(7)$ & $1(2)$ & $1(33)$ & $9(6)$ & $8(2)$ \\
\hline Total reports identifying a pathogen & $94(100)$ & $52(100)$ & $3(100)$ & $149(100)$ & $398(100)$ \\
\hline
\end{tabular}

* Either a single contaminated ingredient was identified or all ingredients in the implicated food belonged to a single food category

$\dagger$ Includes serotypes Berta (1 turkey outbreak), Braenderup (1 chicken outbreak), Enteritidis ( 9 chicken, 2 turkey), Hadar (3 turkey), Heidelberg (7 chicken, 4 turkey), Javiana (1 chicken), Montevideo (1 chicken), Muenchen (1 chicken), Newport ( 3 chicken, 3 turkey), Thompson ( 1 chicken), Typhimurium ( 5 chicken, 5 turkey), and unspecified (10 chicken, 7 turkey)

$\$$ Includes: chemicals, Clostridium botulinum, Cyclospora cayetanensis, Escherichia coli, heavy metals, multiple, other bacterial, other parasites, sapovirus, Shigella sonnei, Streptococcus group A, toxins, Trichinella spiralis, Vibrio spp., and Yersinia enterocolitica.

associated with poultry accounted for more illnesses (9760 of 35622, 27\%) and hospitalizations (565 of $2640,21 \%$ ) than outbreaks caused by any other food category; poultry accounted for the second greatest number of deaths $(22$ of $86,26 \%$ ). In the poultryassociated outbreaks, the mean number of reported illnesses was $35[95 \%$ confidence interval (CI) 25-45], which was not significantly larger than those associated with foods in the other categories (mean $31,95 \%$ CI $25-36)(P=0 \cdot 47)$. Overall, the distributions of age group and sex of patients were similar (all $P \geqslant 0.05$ ) in poultry-associated outbreaks and those associated with other food categories.

\section{Poultry-associated outbreaks with a confirmed pathogen}

Among the 279 poultry-associated outbreaks, 149 $(53 \%)$ had a confirmed pathogen and are the focus of the remainder of the analysis. Salmonella caused the largest number of outbreaks $(64,43 \%)$, followed by C. perfringens (39, 26\%) (Table 1). Other causes were Campylobacter spp. (10, 7\%), norovirus (10, 7\%), Staphylococcus aureus enterotoxin (8, 5\%), Listeria monocytogenes (5, 3\%), Bacillus cereus (4, 3\%), and other agents $(9,6 \%)$. Four serotypes [Enteritidis, 11 outbreaks (17\%); Heidelberg, 11 (17\%); Typhimurium, 10 $(16 \%)$; Newport, $6(9 \%)$ ] caused more than half of the
Salmonella outbreaks. Salmonella outbreaks resulted in the most illnesses $(2614,50 \%)$ and the most hospitalizations $(425,80 \%)$ (Table 2).

A single known location of food preparation was reported for $125(84 \%)$ of these outbreaks. Most were restaurants $(46,37 \%)$, followed by private homes $(31,25 \%)$ and catering facilities $(16,13 \%)$. In restaurant-associated outbreaks, Salmonella (35\%), C. perfringens (26\%), Campylobacter (11\%), and norovirus $(11 \%)$ were the most common aetiologies. In outbreaks in which food was prepared in a private home, Salmonella caused $65 \%$ of outbreaks, and $C$. perfringens caused $26 \%$. C . perfringens caused most of the caterer-associated outbreaks $(56 \%)$.

Information on one or more factors contributing to the outbreak was reported in $120(81 \%)$ outbreaks. In these outbreaks, the most commonly reported contributing factors were food-handling errors $(64 \%)$ and inadequate cooking $(53 \%)$. Among outbreaks caused by Salmonella, inadequate cooking (63\%) and contamination of the uncooked poultry before food preparation $(63 \%)$ were the most commonly reported contributing factors, followed by food-handling errors $(50 \%)$. In $100 \%$ of outbreaks of norovirus infection, investigators reported poor personal hygiene of a food handler as a contributing factor. In outbreaks caused by $C$. perfringens, B. cereus, and $S$. aureus enterotoxin, food-handling errors were the most common contributing factor. 
Table 2. Illnesses, hospitalizations, and deaths in reported poultry-associated* foodborne disease outbreaks with a confirmed pathogen, United States, 1998-2012

\begin{tabular}{llll}
\hline \hline Pathogen & $\begin{array}{l}\text { Illnesses } \\
n(\% \text { of total })\end{array}$ & $\begin{array}{l}\text { Hospitalizations } \\
n(\% \text { of total })\end{array}$ & $\begin{array}{l}\text { Deaths } \\
n(\% \text { of total })\end{array}$ \\
\hline Salmonella enterica & $2614(50)$ & $425(80)$ & $4(20)$ \\
Clostridium perfringens & $1764(33)$ & $8(2)$ & $0(0)$ \\
Norovirus & $191(4)$ & $0(0)$ & $0(0)$ \\
Staphylococcus aureus & $133(3)$ & $19(4)$ & $0(0)$ \\
Listeria monocytogenes & $127(2)$ & $45(8)$ & $16(80)$ \\
Bacillus cereus & $31(1)$ & $1(0)$ & $0(0)$ \\
Campylobacter spp. & $81(2)$ & $5(1)$ & $0(0)$ \\
Other† & $325(6)$ & $28(5)$ & $0(0)$ \\
Total & $5266(100)$ & $531(100)$ & $20(100)$ \\
\hline
\end{tabular}

* Either poultry was identified as the contaminated food or ingredient or all ingredients in the implicated food belonged to the poultry category.

$\dagger$ Includes: Shiga toxin-producing Escherichia coli O157, Shigella sonnei, Vibrio parahaemolyticus, multiple pathogens, and other bacterial pathogens

The 13 outbreaks that involved $>100$ ill persons (range 101-312) accounted for $42 \%$ (2200) of all illnesses in poultry-associated outbreaks. Salmonella caused seven $(54 \%)$ and $C$. perfringens caused six $(46 \%)$. Three of these large outbreaks, including the largest (312 illnesses), occurred in prisons for men; five were restaurant-associated.

\section{Outbreaks by poultry type}

Reports implicated duck in one outbreak and poultry of an unspecified type in two outbreaks; we excluded these three outbreaks from further analyses by poultry type. In the remaining $146(98 \%)$ poultry-associated outbreaks, investigators implicated chicken in 94 outbreaks $(64 \%)$ and turkey in $52(36 \%)$ (Table 1). Although the mean number of illnesses appeared greater in turkey-associated outbreaks than in chicken-associated outbreaks, this finding was not statistically significant (45 vs. 31, $P=0 \cdot 12$ ). Of the largest outbreaks ( $>100$ ill persons), turkey was implicated in seven (54\%) and chicken in six (46\%). Listeria caused a greater percentage of turkey-associated than chicken-associated outbreaks, although this difference was not statistically significant ( $8 \%$ vs. $1 \%, P=0.05$ ); delicatessen meat was the source of all turkey-associated Listeria outbreaks.

Of the 77 chicken-associated outbreaks with a single reported known location of food preparation, $45 \%$ (35 of 77) were associated with food prepared in a restaurant, much greater than the $20 \%$ (9 of 45 ) of turkey-associated outbreaks that were associated with a restaurant $(P=0 \cdot 006)$.

Of the 52 turkey-associated outbreaks, $22(42 \%)$ occurred during November and December (Fig. 1). Chicken-associated outbreaks had a small seasonal peak during the 3-month span from April to June [39 $(41 \%)$ out of 94 reported] (Fig. 2). Although the distribution of pathogens in turkey-associated outbreaks was generally similar across months, $C$. perfringens caused $55 \%$ of turkey-associated outbreaks that occurred during November and December but only $13 \%$ during the other months $(P=0 \cdot 002)$. Improper food handling was a contributing factor more frequently during November and December than during the other months for both turkey-associated outbreaks ( $85 \%$ vs. $45 \%, P=0.01)$ and chicken-associated outbreaks $(100 \%$ vs. $58 \%, P=0.006)$.

In all, 20 deaths were reported in seven outbreaks with a confirmed pathogen and known poultry type. Salmonella caused four outbreaks with one death each (two turkey-associated, two chicken-associated). Listeria caused three outbreaks with 16 deaths - 80\% of all reported deaths - and all were linked to turkey delicatessen meat. The last death in the study period in a Listeria outbreak associated with turkey delicatessen meat was in 2005.

\section{DISCUSSION}

This report highlights the role of poultry as a major source of foodborne outbreaks in the United States. 


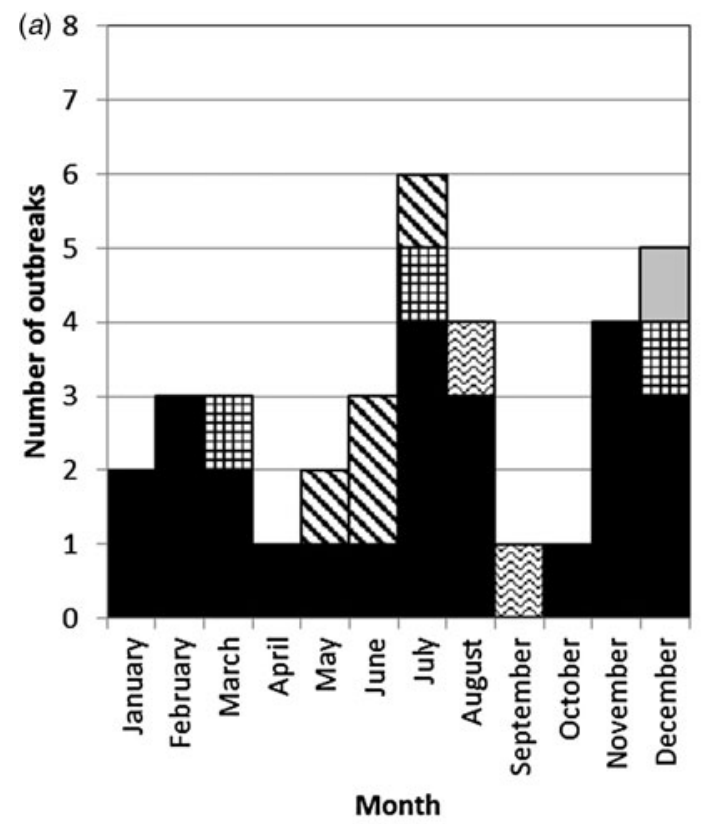

口Other

$\boldsymbol{\Delta}$ Listeria monocytogenes

田 Norovirus

అCampylobacter spp.

- Salmonella enterica

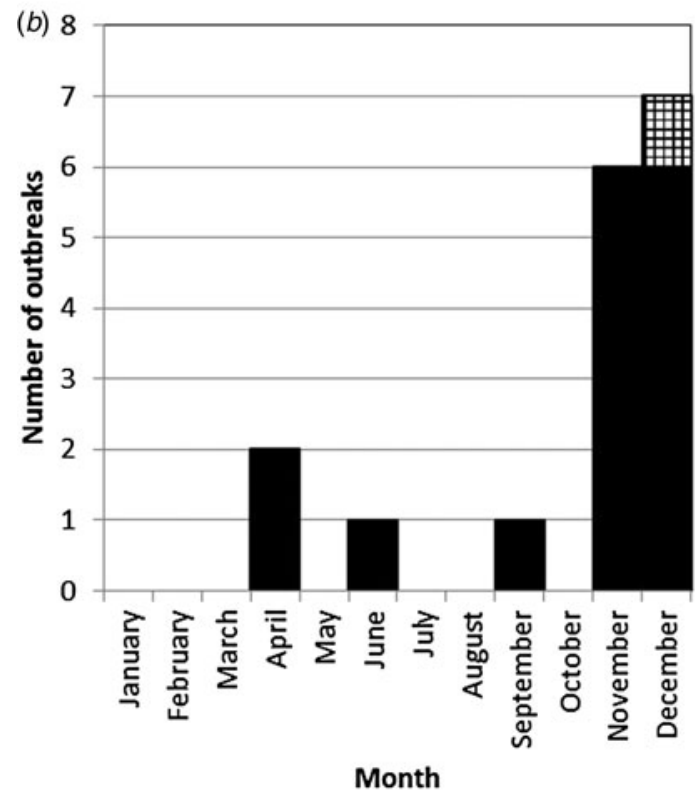

Bacillus cereus

田Staphylococcus aureus

- Clostridium perfringens

Fig. 1. Number of foodborne disease outbreaks with confirmed pathogen associated with turkey, by pathogen and month, United States, 1998-2012. (a) Non-toxin-producing pathogens; (b) toxin-producing pathogens.

With poultry consumption increasing globally, this finding has implications for food safety both within and beyond the United States. From 1998 to 2012, poultry accounted for more reports of foodborne disease outbreaks than any other single food category. Poultry-associated outbreaks accounted for the greatest number of illnesses and hospitalizations and over a quarter of the deaths in outbreaks associated with a single food category. Our analysis of the pathogens, contributing factors, settings, and poultry types associated with foodborne disease outbreaks suggests that breakdowns in food safety at multiple points along the farm-to-fork continuum commonly lead to these outbreaks.

Contamination before food preparation and inadequate cooking were two of the most common contributing factors associated with Salmonella outbreaks. Salmonella is a common contaminant of poultry [5]. In whole chicken carcass testing conducted from 1998 to 2014, cultures of about $4-16 \%$ of carcass rinses in production plants yielded Salmonella [13]. Testing data suggest that contamination of whole 


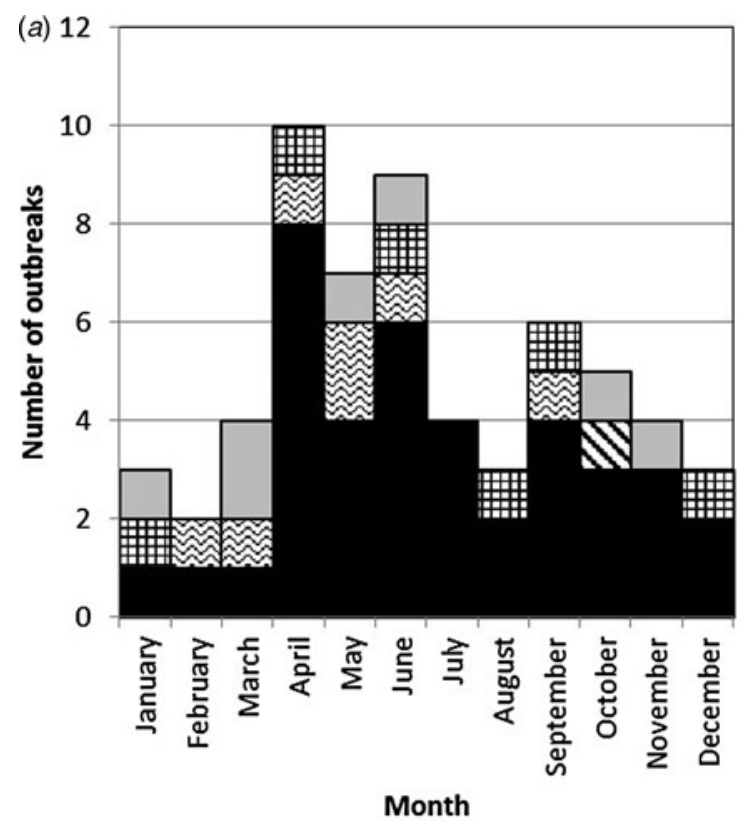

$\square$ Other

వListeria monocytogenes

田Norovirus

Q Campylobacter spp.

- Salmonella enterica

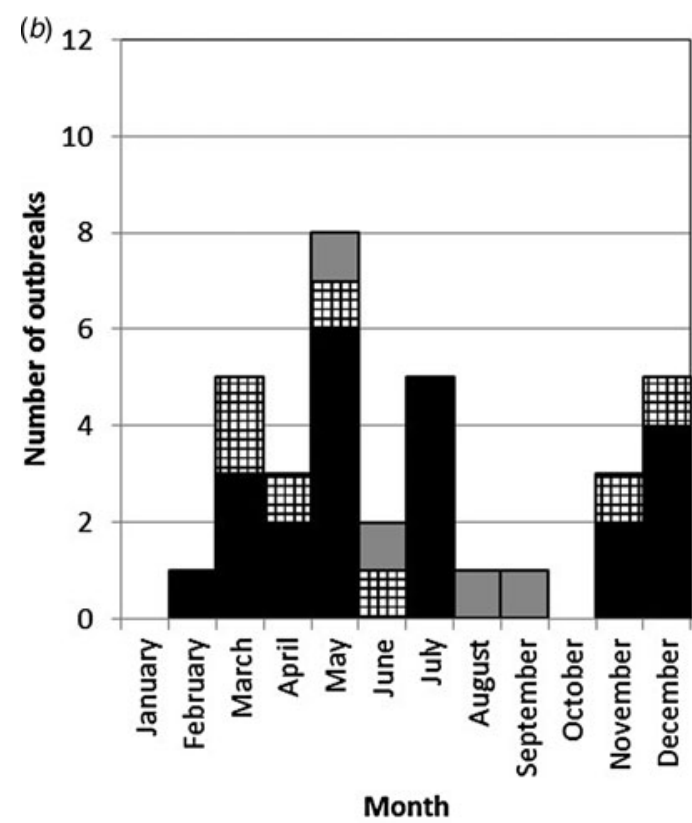

\section{$\square$ Bacillus cereus}

田Staphylococcus aureus

- Clostridium perfringens

Fig. 2. Number of foodborne disease outbreaks with confirmed pathogen associated with chicken, by pathogen and month, United States, 1998-2012. (a) Non-toxin-producing pathogens; (b) toxin-producing pathogens.

chicken carcasses with Salmonella at production decreased between 2002 and 2013 (16.3\% of carcass rinses yielded Salmonella in 2005 vs. $3.9 \%$ in 2013) [13]. Chicken is not usually purchased whole, however [14], and testing of chicken breasts at retail over the same period did not show a similar reduction [8]. Importantly, estimates of chicken consumption more than doubled during 1970-2013 from 37 to $82 \mathrm{lb}$ per capita per year [1]. The U.S. Department of Agriculture (USDA) Food Safety and Inspection
Service (FSIS) conducted a baseline survey of raw chicken parts collected at processing in 2012 and found that the prevalence of Salmonella in samples of pooled chicken parts was $24 \%$ [15]. That same year, Salmonella was found in $18 \%$ of chicken breast samples collected at retail through the NARMS retail meat study [8]. Inadequate cooking, a later step in the farm-to-fork continuum, was also a major contributing factor and was reported in nearly two-thirds of poultry-associated outbreaks. Addressing Salmonella 
contamination in poultry at the farm, during processing, and before food preparation, along with ensuring poultry is adequately cooked before consumption, must continue to be the top priorities in efforts to improve food safety in the United States.

Outbreaks associated with poultry caused by toxinproducing pathogens, most commonly $C$. perfringens, $S$. aureus, and B. cereus, are typically the result of unsafe food handling practices that allow toxin to be formed in cooked food. C. perfringens, S. aureus, and $B$. cereus together accounted for more than one-third of all outbreaks, with $C$. perfringens causing nearly a third of all illnesses, indicating that lapses in safe food handling practices were common in poultry-associated outbreaks. For caterers, the prominence of $C$. perfringens as a cause of poultryassociated outbreaks emphasizes the importance of proper holding times and temperature control, especially for foods that are prepared in advance and held for relatively long periods before or while being served $[16,17]$. C. perfringens caused nearly half of the 13 largest poultry-associated outbreaks, which themselves represented only $9 \%$ of all poultryassociated outbreaks but accounted for nearly half of all illnesses. Especially large $C$. perfringens outbreaks occurred in institutional settings, where other toxin-mediated outbreaks also are common $[18,19]$. Lack of knowledge about safe food preparation techniques contributes to suboptimal practices [20, 21]. Improving holding practices for poultry-containing foods, especially in large congregate settings, could reduce markedly the number of outbreak-associated illnesses.

Seasonal consumption patterns and lapses in safe food preparation practices were also important drivers of poultry-associated outbreaks. Nearly half of all turkey-associated outbreaks occurred during the November (Thanksgiving) and December (Christmas) holiday season in the United States, when turkey is traditionally eaten. According to quarterly USDA data from 1998 to 2012 on turkey meat 'disappearance', $31 \%$ of turkeys are eaten in the last 3 months of the year [1]. As $42 \%$ of all turkey-associated outbreaks occurred in November and December, factors beyond increased consumption might contribute to turkey-associated outbreaks in those two months. Investigators more commonly identified $C$. perfringens as the cause of turkey-associated outbreaks during these two months than the rest of the year, likely reflecting food-handling errors. The importance of safe handling practices, including proper holding, cooling, storage, and reheating of food, is highlighted by the increased number of outbreaks with reported improper food-handling practices during the holiday season for both turkey and chicken.

Contamination of food by a food handler can also cause poultry-associated outbreaks. Norovirus typically contaminates food during handling by infected persons. Norovirus caused about 1 in 9 restaurant-associated outbreaks with a confirmed pathogen, and because health department access to norovirus diagnostics were often limited in the early years of our study, it likely caused even more of the outbreaks with a suspected pathogen. Infected food handlers who do not wash hands well after defecating, whether asymptomatic or ill, can contaminate many dishes, infecting many people. Persons with gastrointestinal illness should not handle food for others, yet food handlers often work when ill, especially when they do not have paid sick leave [22, 23].

The true contribution of contaminated poultry to outbreaks and to overall foodborne illness is likely even higher than what we report, for several reasons. When a food contains several ingredients, and the epidemiological or laboratory evidence implicates only the food and not the contaminated ingredient, poultry will not be implicated, even if it is contaminated. Moreover, pathogens on poultry can crosscontaminate other foods during food preparation [24]. Such outbreaks would be attributed to the other (cross-contaminated) food or no food (crosscontamination of several foods can make identification of the source impossible), not to poultry. In fact, one risk assessment suggested that cross contamination of ready-to-eat foods from contaminated poultry might cause more illnesses than inadequate cooking of poultry [25]. Antibiotic-resistant salmonella has caused recent poultry-associated outbreaks and high rates of hospitalization $[9,26]$, heightening the urgency of implementing focused interventions.

Several general limitations of foodborne disease outbreak surveillance exist. Restaurant- and catererassociated outbreaks are more likely to be investigated and reported than outbreaks associated with other settings [27]. Therefore, our finding that these settings account for half of poultry-associated outbreaks probably reflects some degree of surveillance bias. Salmonella might be overrepresented, compared with toxin-mediated illnesses, because of the longer duration of symptoms, more severe illness in salmonellosis, and microbiological diagnosis of Salmonella infection is more common. In many outbreaks, a pathogen is not identified, either because clinical samples are not collected, food has already been thrown 
away by the time an investigation is initiated, an epidemiological link cannot be found, or because of other factors [28]. National foodborne disease outbreak surveillance is passive, leading to underreporting. In addition, we could not meaningfully assess trends, given the relatively limited number of outbreaks during the study period.

Regulatory agencies in the United States have implemented measures to reduce poultry-associated illness. In 1996, FSIS issued the Pathogen Reduction; Hazard Analysis and Critical Control Point Systems Final Rule, which set standards for Salmonella for whole chickens, ground turkey, and ground chicken for poultry processing establishments. In 2006, FSIS implemented baseline prevalence performance standards for Salmonella for turkey carcasses [29] and, in 2011, strengthened standards for whole chicken samples yielding Salmonella and implemented a new standard for Campylobacter for whole chickens [30]. In addition, FSIS strengthened standards for pooled turkey carcass samples yielding Salmonella and Campylobacter in 2011 [30]. In 2015, FSIS proposed new performance standards for Salmonella and Campylobacter on chicken parts and not-ready-to-eat comminuted poultry [31]. Spurred by multiple outbreaks of Listeria infection associated with turkey delicatessen meat [32, 33], USDA initiated regulations in 2003 that addressed this issue [34]; from 2003 to 2013, only one outbreak of listeriosis associated with poultry delicatessen meat was reported, which occurred in 2005 [35, 36]. The U. S. Food and Drug Administration (FDA) also has helped to set a model for food safety practices and regulations in restaurants and institutional settings through its Food Code [37], and some states have implemented restaurant manager food-safety training [38]. In addition, US federal government-wide efforts, such as the Department of Health and Human Services' food safety website (www.foodsafety.gov) and the Ad Council's campaigns with the USDA on food safety during the Thanksgiving holidays, educate consumers about food safety practices.

Understanding the pathogens, settings, contamination factors, and poultry types leading to poultryassociated outbreaks helps identify lapses in food safety practices along the farm-to-fork continuum and helps target interventions needed to decrease illnesses associated with poultry in the United States. These sources and circumstances of food contamination leading to illness might be common across countries, and the fast-growing consumption of poultry worldwide places urgency on identifying additional sources and circumstances. Successful efforts to curb poultry-associated illnesses could have a large impact on the overall incidence of foodborne illnesses in the United States and beyond.

\section{SUPPLEMENTARY MATERIAL}

For supplementary material accompanying this paper visit https://doi.org/10.1017/S0950268816002375.

\section{ACKNOWLEDGEMENTS}

The authors acknowledge Patricia M. Griffin and L. Hannah Gould (both of Centers for Disease Control and Prevention, Atlanta, GA, USA) for their valuable contributions to this manuscript. This research received no specific grant from any funding agency, commercial or not-for-profit sectors.

The opinions expressed by authors contributing to this journal do not necessarily reflect the opinions of the Centers for Disease Control and Prevention or the institutions with which the authors are affiliated.

\section{DECLARATION OF INTEREST}

None.

\section{REFERENCES}

1. Livestock and Poultry: World Markets and Trade. Washington, DC: US Department of Agriculture, Foreign Agricultural Service, 2016 (http://apps.fas.usda. gov/psdonline/circulars/livestock_poultry.pdf). Accessed 10 July 2016.

2. Daniel CR, et al. Trends in meat consumption in the USA. Public Health Nutrition 2011; 14: 575-583.

3. Scallan E, et al. Foodborne illness acquired in the United States-major pathogens. Emerging Infectious Diseases 2011; 17: 7-15.

4. Friedman CR, et al. Risk factors for sporadic Campylobacter infection in the United States: A casecontrol study in FoodNet sites. Clinical Infectious Diseases 2004; 38 (Suppl. 3): S285-296.

5. Guo C, et al. Application of Bayesian techniques to model the burden of human salmonellosis attributable to U.S. food commodities at the point of processing: adaptation of a Danish model. Foodborne Pathogens and Disease 2011; 8: 509-516.

6. Kimura AC, et al. Chicken consumption is a newly identified risk factor for sporadic Salmonella enterica serotype Enteritidis infections in the United States: a case-control study in FoodNet sites. Clinical Infectious Diseases 2004; 38 (Suppl. 3): S244-252.

7. Painter JA, et al. Attribution of foodborne illnesses, hospitalizations, and deaths to food commodities by 
using outbreak data, United States, 1998-2008. Emerging Infectious Diseases 2013; 19: 407-415.

8. NARMS Integrated Report: 2012-2013. The National Antimicrobial Resistance Monitoring System: Enteric Bacteria. Silver Spring, MD: U.S. Department of Health and Human Services, Food and Drug Administration, 2015 (http://www.fda.gov/downloads/ AnimalVeterinary/SafetyHealth/AntimicrobialResistance/ NationalAntimicrobialResistanceMonitoringSystem/UCM 453398.pdf). Accessed 25 March 2016.

9. Centers for Disease Control and Prevention. Multistate outbreak of multidrug-resistant Salmonella Heidelberg infections linked to foster farms brand chicken (final update), 2014 (http://www.cdc.gov/salmonella/heidelberg10-13/index.html). Accessed 31 July 2014.

10. Bryan FL, Guzewich JJ, Todd WCD. Surveillance of foodborne disease III. Summary and presentation of data on vehicles and contributory factors; their value and limitations. Journal of Food Protection 1997; 60: 701-714.

11. Centers for Disease Control and Prevention. Guide to confirming a diagnosis in foodborne disease, 2009 (http://www.cdc.gov/foodsafety/outbreaks/investigatingoutbreaks/confirming_diagnosis.html). Accessed 16 February 2011.

12. Painter JA, et al. Recipes for foodborne outbreaks: a scheme for categorizing and grouping implicated foods. Foodborne Pathogens and Disease 2009; 6: 1259-1264.

13. U.S. Department of Agriculture, Food Safety and Inspection Service. Progress Report on Salmonella and Campylobacter Testing of Raw Meat and Poultry Products, 1998-2014 (http://www.fsis.usda.gov/wps/wcm/ connect/7b9ba8cd-de00-4d8d-8cf7-7cfbe24236f7/ProgressReport-Salmonella-Campylobacter-CY2014.pdf?MOD= AJPERES). Accessed 21 August 2015.

14. National Chicken Council. How broilers are marketed. (http://www.nationalchickencouncil.org/about-the-industry/ statistics/how-broilers-are-marketed/). Accessed 6 April 2014.

15. U.S. Department of Agriculture. The Nationwide Microbiological Baseline Data Collection Program: Raw Chicken Parts Survey, January 2012-August 2012. (http://www.fsis.usda.gov/wps/wcm/connect/a9837fc80109-4041-bd0c-729924a79201/Baseline_Data_Raw_ Chicken_Parts.pdf?MOD=AJPERES). Accessed 5 April 2014.

16. Bennett SD, Walsh KA, Gould LH. Foodborne disease outbreaks caused by Bacillus cereus, Clostridium perfringens, and Staphylococcus aureus - United States, 19982008. Clinical Infectious Diseases 2013; 57: 425-433.

17. Grass JE, Gould LH, Mahon BE. Epidemiology of foodborne disease outbreaks caused by Clostridium perfringens, United States, 1998-2010. Foodborne Pathogens and Disease 2013; 10: 131-136.

18. Centers for Disease Control and Prevention. Clostridium perfringens infection among inmates at a county jail Wisconsin, August 2008. Morbidity and Mortality Weekly Report 2009; 58: 138-141.
19. Cieslak PR, et al. Preventable disease in correctional facilities. Desmoteric foodborne outbreaks in the United States, 1974-1991. Archives of Internal Medicine 1996; 156: $1883-1888$.

20. Abbot JM, et al. Comparison of food safety cognitions and self-reported food-handling behaviors with observed food safety behaviors of young adults. European Journal of Clinical Nutrition 2009; 63: 572-579.

21. Roseman M, Kurzynske J. Food safety perceptions and behaviors of Kentucky consumers. Journal of Food Protection 2006; 69: 1412-1421.

22. Carpenter LR, et al. Food worker experiences with and beliefs about working while ill. Journal of Food Protection 2013; 76: 2146-2154.

23. Sumner S, et al. Factors associated with food workers working while experiencing vomiting or diarrhea. Journal of Food Protection 2011; 74: 215-220.

24. Verhoeff-Bakkenes L, et al. Quantification of Campylobacter jejuni cross-contamination via hands, cutlery, and cutting board during preparation of a chicken fruit salad. Journal of Food Protection 2008; 71: 1018-1022.

25. Luber P. Cross-contamination versus undercooking of poultry meat or eggs - which risks need to be managed first? International Journal of Food Microbiology 2009; 134: 21-28.

26. Centers for Disease Control and Prevention. Outbreak of Salmonella Heidelberg infections linked to a single poultry producer-13 states, 2012-2013. Morbidity and Mortality Weekly Report 2013; 62: 553-556.

27. Batz MB, et al. Attributing illness to food. Emerging Infectious Diseases 2005; 11: 993-999.

28. Murphree R, et al. Characteristics of foodborne disease outbreak investigations conducted by Foodborne Diseases Active Surveillance Network (FoodNet) sites, 2003-2008. Clinical Infectious Diseases 2012; 54 (Suppl. 5): S498-503.

29. Food Safety and Inspection Service. Salmonella verification sample result reporting: agency policy and use in public health protection. Federal Register 2006; vol. 71, no. 38.

30. Food Safety and Inspection Service. New performance standards for Salmonella and Campylobacter in young chicken and turkey slaughter establishments: response to comments and announcement of implementation schedule. Federal Register 2011; vol. 76, no. 54.

31. Food Safety and Inspection Service. Changes to the Salmonella and Campylobacter verification testing program: proposed performance standards for Salmonella and Campylobacter in not-ready-to-eat comminuted chicken and turkey products and raw chicken parts and related agency verification procedures and other changes to agency sampling. Federal Register 2015; vol. 80, no. 16 .

32. Gottlieb SL, et al. Multistate outbreak of listeriosis linked to turkey deli meat and subsequent changes in US regulatory policy. Clinical Infectious Diseases 2006; 42: 29-36. 
33. Olsen SJ, et al. Multistate outbreak of Listeria monocytogenes infection linked to delicatessen turkey meat. Clinical Infectious Diseases 2005; 40: 962-967.

34. Food Safety and Inspection Service. Control of Listeria monocytogenes in ready-to-eat meat and poultry products, final rule. Federal Register 2003; vol. 68, no. 109.

35. Centers for Disease Control and Prevention. Surveillance for foodborne disease outbreaks - United States, 20092010. Morbidity and Mortality Weekly Report 2013; 62: $41-47$.
36. Cartwright EJ, et al. Listeriosis outbreaks and associated food vehicles, United States, 1998-2008. Emerging Infectious Diseases 2013; 19: 1-9; quiz 184.

37. United States Public Health Service, Food and Drug Administration. Food code, 2009 (http://www.fda.gov/ downloads/Food/FoodSafety/RetailFoodProtection/Food Code/FoodCode2009/UCM189448.pdf). Accessed 16 October 2012.

38. Cates SC, et al. Certified kitchen managers: do they improve restaurant inspection outcomes? Journal of Food Protection 2009; 72: 384-391. 